\title{
Evolución en el uso de las tecnologías de la información y comunicación (TIC) y competencias de los docentes de la Universidad Autónoma de Chihuahua (México), durante la pandemia
}

\author{
Ana M. Arras-Vota ${ }^{1}$, José L. Bordas-Beltrán ${ }^{2 *}$, Damián A. Porras-Flores ${ }^{1}$ y María del Carmen Gutiérrez Diez ${ }^{2}$ \\ (1) Facultad de Ciencias Agrotecnológicas, Universidad Autónoma de Chihuahua. Chihuahua, México. \\ (correo-e: aarras@uach.mx; correo-e: dporras@uach.mx \\ (2) Facultad de Contaduría y Administración, Universidad Autónoma de Chihuahua. Chihuahua, México. \\ (correo-e: jbordas@uach.mx; correo-e: cgutierr@uach.mx)
}

* Autor a quien debe ser dirigida la correspondencia

Recibido Jun. 9, 2021; Aceptado Jul. 29, 2021; Versión final Ago. 21, 2021, Publicado Dic. 2021

\begin{abstract}
Resumen
El propósito de la presente investigación fue comparar la percepción que tienen los docentes de la Universidad Autónoma de Chihuahua de México sobre el uso de las tecnologías de la información y comunicación (TIC) durante dos periodos comprendidos en el año 2020. Se utilizaron los métodos analítico- sintético y teóricodeductivo y la investigación se realizó a partir de un estudio de caso. Para seleccionar a los participantes se empleó el muestreo probabilístico y para recoger información de campo se realizaron encuestas. El instrumento de medición fue validado con un Alfa de Cronbach superior a 0.8. Se concluyó que los docentes de la universidad percibieron haber incrementado el dominio de sus competencias en general. Se observó que el uso de la plataforma institucional incrementó significativamente. La universidad debe seguir implementado estrategias que permitan a sus docentes continuar evolucionando durante la pandemia del COVID-19 con el fin de continuar desarrollando entornos de aprendizaje más amigables e intuitivos para los alumnos, entornos que permanecerán después de esta crisis.
\end{abstract}

\section{Evolution in the use of information and communication technologies (ICT) and competences of the teachers of the Autonomous University of Chihuahua (Mexico), during the pandemic}

\begin{abstract}
The purpose of this research was to compare the perception that teachers of the Autonomous University of Chihuahua of Mexico have on the use of information and communication technologies (ICT) during two periods in 2020. Analytical - synthetic and theoretical - deductive methods were used and was carried out from a case study. The techniques applied to select participants was probability sampling and to gather field information, surveys were conducted. The measuring instrument was validated with a Cronbach's Alpha greater than 0.8 . It was concluded that the university teachers perceived that they increased their skills in general, and it was also observed that the use of the institutional platform significantly increased its use. The university must continue implementing strategies that allow professors to continue evolving during the COVID-19 pandemic in order to continue developing more user-friendly and intuitive learning environments for students, environment that will remain even after this crisis.
\end{abstract}




\section{INTRODUCCIÓN}

México, al igual que muchos otros países del mundo, dio respuesta al llamado de emergencia mundial, el 14 de marzo de 2020, cuando la Secretaría de Educación Pública del Gobierno, informó a la comunidad en general las medidas de prevención para el sector educativo en toda la nación, las cuales acataron las disposiciones de la Organización Mundial para la Salud (OMS) (Cervantes et al., 2020), lo que implicó el cierre de los centros educativos, en un contexto de cambio no planificado que demandó la adaptación de procesos digitales al diseño instructivo (Santiago et al., 2021); esto, con el fin de reducir la transmisión del virus en la amplia mayoría de los países del mundo (Ruiz, 2020), derivó en un tránsito inmediato de la educación presencial a la virtual; el profesorado pasó a trabajar con los estudiantes a través de diversas plataformas y tecnologías y el alumnado quedó confinado en sus casas; lo que significó y sigue significando, un desafío para los actores sociales, desde los estudiantes y docentes, hasta los directivos.

En pocos días se había orquestado un sistema alternativo de aprendizaje a distancia, sin precedentes similares nunca vistos, y muy estrechamente apoyado en la autogestión y la autorregulación (Muñoz et al., 2020), lo que supuso la pérdida de los espacios físicos de encuentro con otros estudiantes y con los docentes, esto deriva en la sensación de haber perdido la escuela, por la traslación realizada de una modalidad a la otra, de allí la importancia de analizar las condiciones en las que el sistema escolar, los alumnos y los profesores han enfrentado esta situación (Díaz-Barriga, 2020), en la que la institución educativa y el hogar se convierten en uno solo y, de acuerdo con Ruiz (2020), todo este reto puede llevar a dejar de reconocer que el mayor riesgo es dejar de contemplar las condiciones de emergencia (sanitarias, sociales, psicológicas y tecnológicas) generadas por la pandemia del Covid-19, ya que ello afectó y sigue incidiendo en la educación y sus implicancias formativas para el desarrollo humano.

En el actual escenario, los docentes de todos los niveles educacionales, debieron forzosamente incorporar nuevas herramientas, más allá del uso de las TIC, o algún software, sin que necesariamente mediara preparación alguna, la pandemia los obligó a salir del papel, de la clase presencial, de las evidencias, y de todos los instrumentos de evaluación formativos, entre otras prácticas, para adentrarse en las plataformas de educación a distancia, sin diferenciar entre profesores recién egresados, de aquellos que esperan su retiro (Arriagada, 2020). Todo lo cual ha llevado a encarar el reto que se vive en los espacios educativos, donde los profesores han reivindicado la importancia de actuar como un auténtico equipo, liderando valores y creando climas de esfuerzo y motivación con el propósito de sostener los centros escolares como comunidades educativas.

Además, los alumnos y sus familias se han visto con la obligación de dotarse de herramientas e instrumentos que les permitan proseguir con el temario y las tareas escolares desde casa; $y$, singularmente, por parte de los profesionales de la educación para planificar lo apropiado para seleccionar cómo los estudiantes podían utilizar mejor su entorno y de qué recursos disponían para mantener la continuidad del aprendizaje durante el cierre de los centros escolares (Muñoz et al., 2020), durante el Covid-19, lo cual representa, sin duda, un cambio estructural en la educación universitaria y en la forma de impartir docencia (Torrecillas, 2020), de allí la importancia de realizar estudios que evalúen los resultados de esta contingencia sanitaria para la educación y la vida escolar respecto a las condiciones de trabajo de los actores de la educación y su experiencia en entornos virtuales de aprendizaje (Cervantes et al., 2020), en dos semestres el primero en el tránsito inmediato $y$, posteriormente, en el segundo semestre del año 2020 con el objetivo de analizar comparativamente la evolución en el uso de las TIC y competencias de los docentes de la Universidad Autónoma de Chihuahua $(\mathrm{UACH})$, después de recibir capacitación para transitar de la educación presencial a la virtual, al tiempo que generen información sobre las formas en que se han visto afectados los colectivos de académicos ante las nuevas condiciones que prevalecen en el entorno educativo, social y económico.

El tránsito repentino entre la modalidad presencial y la virtual dejó vacíos en cuanto al acceso y dominio de las Tecnologías de Información y Comunicación (TIC), aspectos que se consideran como barreras tecnológicas, a las que se agregan la económica (no tienen acceso a Internet por no contar con recursos para hacerlo) y material (no cuentan con computadora de uso personal) (Quintana, 2020), las primeras se habrán de subsanar conforme las universidades y centros de estudio van gestando las condiciones tecnológicas y de competencias en TIC de estudiantes y docentes, las cuales se refieren a: "... las habilidades, capacidades y destrezas requeridas para utilizar las herramientas tecnológicas y los medios digitales" (Martzoukou et al., 2020, 1414), a través de la gestión y uso de las TIC, solución de problemas, análisis crítico de contenidos, así como la construcción y socialización ética del conocimiento.

Las competencias en TIC se han clasificado como básicas, de aplicación, profundización, de trabajo colaborativo, éticas y de aprendizaje permanente. Las competencias básicas implican navegar, buscar, clasificar y comunicar a través del uso de tecnologías (Maryuningsih et al., 2019). Las competencias de aplicación se refieren al uso efectivo y productivo de los sistemas de TIC (Infante et al., 2019). Un estudiante puede considerarse competente en el uso de la información cuando es capaz de determinar la naturaleza y 
el nivel de la información necesaria (Jaramillo et al., 2011). Así mismo, una persona tiene competencias más profundas cuando puede resolver problemas, crear trabajos originales, planificar, organizar, evaluar, sintetizar y utilizar información de diversos medios, así como actividades de procesamiento y comunicación relacionadas con un proyecto en particular (Infante et al., 2019). Esta construcción implica análisis y toma decisiones sobre el contenido de los mensajes obtenidos a través de las TIC, lo que significa, según Jaramillo et al. (2011), que Él / Ella puede utilizar la información de manera efectiva para lograr un propósito específico, como la construcción de conocimiento, el cual implica al pensamiento crítico, que presupone observar, identificar problemas y cuestionamientos para guiar una investigación y analizar información desde diferentes perspectivas con el propósito de explorar soluciones alternativas a los planteamientos realizados (Infante et al., 2019).

La construcción del conocimiento y el pensamiento crítico se pueden generar en una comunidad de aprendizaje, la cual es una entidad de prácticas compartidas entre sus miembros, son espacios formales e informales en los que los actores pueden intercambiar ideas, acciones y actuaciones sobre el trabajo conjunto, (Eirín, 2018), estas comunidades van de la mano con las competencias de trabajo colaborativo, las cuales involucran una interacción entre dos o más personas, quienes deliberan sobre “...dos o más temas para construir aprender a través de la discusión, la reflexión y la toma de decisiones sobre recursos de información (Galindo et al., 2013), lo que significa que existe una interacción entre "... dos o más temas para construir y aprender a través de la deliberación, la reflexión y la toma de decisiones sobre recursos de información (Galindo et al., 2013). En este proceso, el pensamiento crítico también es un activo valioso, que igualmente se entrelaza con el aprendizaje a lo largo de la vida, el cual cambia el enfoque de una visión que implica aprender a aprender centrada en el sujeto en formación, con el fin de mejorar en sus procesos de instrucción, lo cual involucra procesos formales e informales que le permiten mejorar sus habilidades y competencias a lo largo de su vida (Cendon, 2018).

Considerar la información disponible para las personas a través de las tecnologías las ubica en una disyuntiva entre el ser y el deber ser en el uso de aquélla, de allí la importancia de considerar la ética en el manejo de los datos. La ética se concibe como un proceso (Lomborg, 2012), una serie de lineamientos que influyen en el comportamiento de los seres humanos en sociedad los cuales les permiten proteger y cumplir sus derechos y los de otros (Marshall, 1999), en ese sentido las competencias éticas en el uso de las TIC, significan que una persona comprende y está entrenada formalmente para reconocer los derechos del autor y citar su trabajo, con el fin de prevenir el plagio (Olivia et al., 2019).

Una vez que se ha planteado la transición del modelo educativo presencial a uno en línea, las competencias en TIC son factor determinante para el avance en la construcción del aprendizaje, en este caso en el contexto de una universidad pública en México, a saber, UACH en dos momentos, al inicio de la pandemia y, luego, en el segundo semestre del 2020. En esta investigación el análisis de competencias, efectos del Covid-19 en la familia y desafíos enfrentados por los docentes, se consideró desde la perspectiva de la percepción, la cual se define como "... uno de los procesos cognitivos, una forma de conocer el mundo, a través de la información que aquella entrega y a partir de las experiencias de quienes perciben (Moreno et al., 2013), es la opinión de los profesores y estudiantes sobre el nivel de dominio (Infante et al., 2019), lo que comprenden sobre sus competencias en TIC (Zambrano et al., 2018).

Esta pregunta eje lleva a plantear el objetivo de comparar la percepción de los docentes de la UACH entre el primer y del segundo semestre del 2020 sobre: Esta investigación, producto del proyecto institucional "Desafíos que enfrentan estudiantes y docentes en e-educación universitaria ante COVID-19", derivada del tránsito de la educación presencial a la virtual y a partir de haber recibido, como parte de las estrategias académicas de la $\mathrm{UACH}$, capacitación, se ha planteado la siguiente pregunta eje: ¿Existen diferencias significativas entre la percepción del primer y del segundo semestre del 2020 de los docentes de la UACH, en torno a 1) los efectos del Covid-19 en su familia, sus 2) competencias básicas, 3) competencias de aplicación, 4) competencias de profundización, 5) competencias de trabajo colaborativo, 6) competencias de aprendizaje para toda la vida y 7) competencias éticas?

\section{METODOLOGÍA}

Esta investigación utilizó los métodos: analítico - sintético y teórico - deductivo y se realizó a partir de un estudio de caso, "el cual contribuye a ampliar y profundizar el conocimiento con respecto a individuos y grupos, así como organizaciones y fenómenos relacionados" (Yin, 2009 p. 18); de forma aplicada y de modo descriptivo ya que explica las características de los profesores con respecto a la percepción de sus competencias digitales y efectos de la pandemia en el entorno familiar. Así mismo, el estudio es de naturaleza cuantitativa, porque recogió los datos a través de escalas y preguntas cerradas, además, es una investigación longitudinal, puesto que se realizó en dos períodos de tiempo específicos, a saber, los semestres de enero a junio y agosto diciembre de 2020. 


\section{Técnicas}

Como técnicas de acopio de la información se utilizaron la documental (bibliografía) y estadística, así como la encuesta por red o dispositivos móviles. La encuesta fue integrada por 14 ítems que se referían a la percepción de los profesores sobre el desarrollo de indicadores de competencias en TIC y por preguntas cerradas sobre los principales desafíos enfrentados en la pandemia y por pasar de la modalidad presencial a la virtual durante la contingencia sanitaria - Covid-19 - a la que se enfrentan actualmente las Instituciones de Educación Superior, entre ellas la universidad objeto de este estudio.

Como punto de partida se utilizó el estudio de las habilidades en TIC en las universidades de Salamanca, España, Veracruzana y Autónoma de Chihuahua (Arras-Vota et al., 2011). Cabe señalar que, para validar el instrumento, éste se envió a pares académicos y, posteriormente, se aplicó una prueba piloto, la retroalimentación vertida por estos grupos condujo a la fase final de elaboración de la encuesta que se aplicó, la cual inicia con un consentimiento informado regido por tres principios éticos.

\section{Procedimientos}

Las encuestas fueron distribuidas mediante un recopilador de datos perteneciente a Google on-line GoogleForms y la información recabada se analizó con el programa SPSS (v.22.0). Se llevó a cabo un análisis de datos cuantitativos a través de estadística descriptiva. Así mismo, se utilizó, una de las pruebas más usadas para medir el grado de confiabilidad de la escala: el coeficiente de alfa de Cronbach (Landero et al., 2006). En este trabajo se obtuvieron las siguientes calificaciones para el periodo comprendido de enero a junio $(\alpha=.814)$, y para el comprendido de agosto a diciembre $(\alpha=.820)$, siendo superior a .8 en ambas situaciones por lo que se considera de acuerdo con Frías (2014), aceptable. Además, en la Figura 1 se muestra una imagen que refleja las etapas de la investigación que se llevaron a cabo.

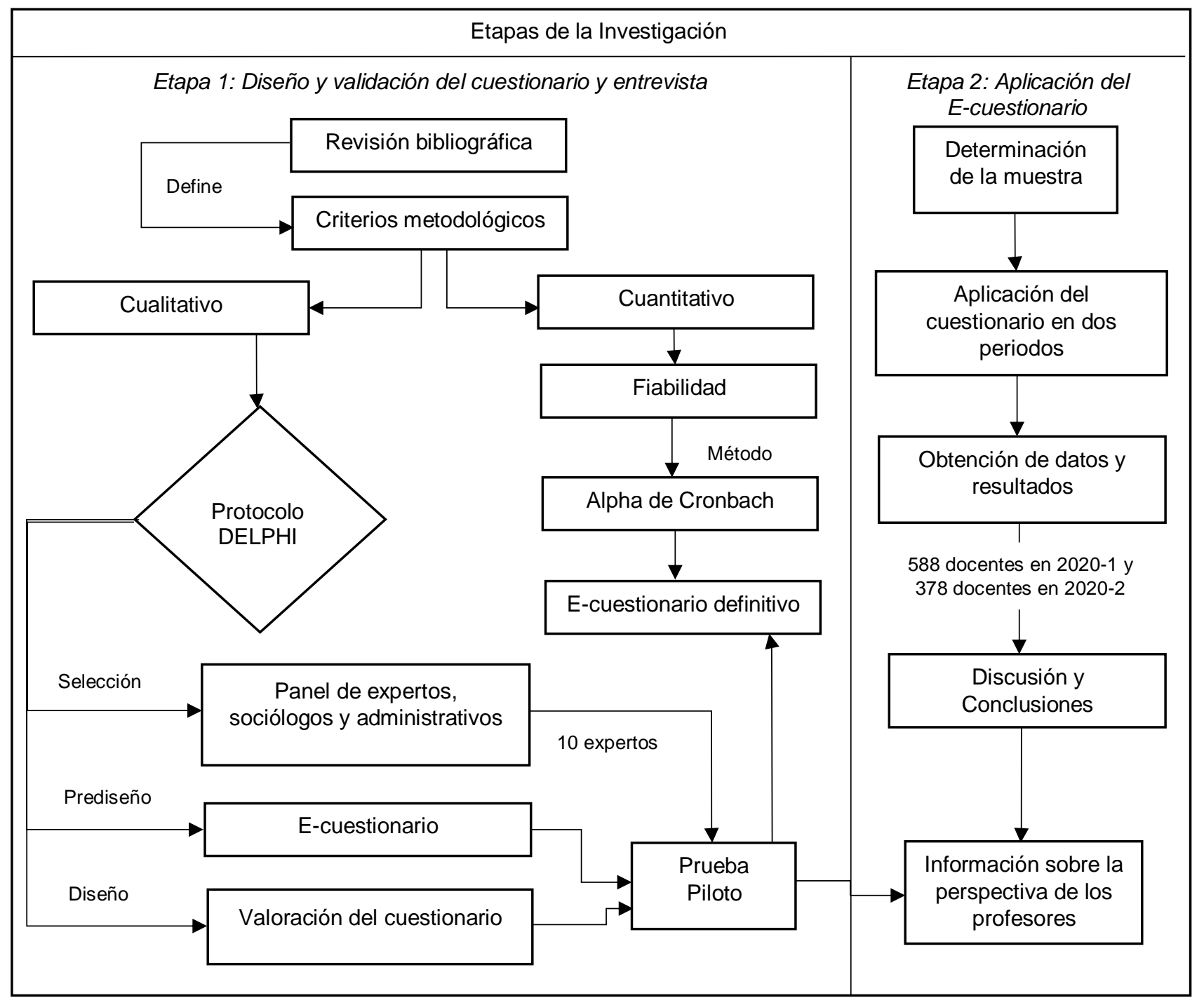

Fig. 1: Es de su agrado impartir clases en modalidad virtual 


\section{Universo de estudio}

El universo de estudio estuvo integrado por docentes de la $\mathrm{UACH}$, la cual se fundó el 8 de diciembre de 1954 y en 1968 logró su autonomía (Universidad Autónoma de Chihuahua, 2020). La UACH (UACH), se encuentra integrada por 15 facultades con 2935 docentes de licenciatura y posgrado; oferta programas educativos a nivel licenciatura y posgrado en 8 ramas del conocimiento tanto en modalidad virtual como presencial.

\section{Muestras}

Para la obtención de las muestras, cuya composición se describe en las tablas 1, 2 y 3, se utilizó la siguiente fórmula para poblaciones finitas:

$n=\frac{z^{2} p q N}{E^{2} N+z^{2} p q}$

La selección fue probabilística, estratificada con afijación proporcional, garantizando la representatividad de la muestra de la población objeto de estudio, la muestra se representa un $95 \%$ de confianza y se integró como se plantea en la tabla 1. La caracterización de las muestras por periodo se resume en la tabla 2 y 3 respectivamente. Las muestras en los dos periodos analizados no presentan diferencias porcentuales importantes, las muestras en ambos periodos se mantienen homogéneas. Cabe señalar que los rangos de edad de los docentes permanecieron en la misma proporción en los dos periodos, a saber, donde el perfil mayoritario de la muestra es entre 31 y 40 años de edad; siendo las edades predominantes de entre 31 y 60 años de edad.

Tabla 1: Tamaño de la muestra

\begin{tabular}{|c|c|c|c|}
\hline & Año & Muestra & Muestra recabada \\
\hline \multirow{3}{*}{ Docentes } & $2020 / 1$ & 340 & 588 \\
\cline { 2 - 4 } & $2020 / 2$ & 340 & 378 \\
\hline
\end{tabular}

Tabla 2: Perfil de la muestra de docentes, por sexo, en los periodos de enerojunio y agosto-dic 2020

\begin{tabular}{|c|c|c|c|}
\hline & Periodo & Mujeres & Hombres \\
\hline \multirow{2}{*}{ Docentes } & $2020 / 1$ & $44.6 \%$ & $44.70 \%$ \\
\cline { 2 - 4 } & $2020 / 2$ & $55.4 \%$ & $55.3 \%$ \\
\hline
\end{tabular}

Tabla 3: Rangos de edad de la muestra de docentes

\begin{tabular}{|c|c|c|}
\hline Población & Porcentaje & Rangos de edad \\
\hline \multirow{4}{*}{ Docentes } & $8.6 \%$ & Entre 20 y 30 años \\
\cline { 2 - 3 } & $35.1 \%$ & Entre 31 y 40 años \\
\cline { 2 - 3 } & $27.5 \%$ & Entre 41 y 50 años \\
\cline { 2 - 3 } & $20.1 \%$ & Entre 51 y 60 años \\
\cline { 2 - 3 } & $8.6 \%$ & 61 o más años \\
\hline
\end{tabular}

\section{RESULTADOS}

A continuación, se presentan los resultados de la percepción de los docentes de la UACH en torno a la forma como consideran la pandemia afectó a su familia lo que se puede observar en la tabla 4, así como las dificultades enfrentadas, de las cuales se incluye el comparativo entre el primer y segundo semestre de 2020 en la tabla 5.

Tabla 4: Afectación en su familia por parte de la pandemia derivada por el COVID-19

\begin{tabular}{|l|c|c|}
\hline & $2020 / 1$ & $2020 / 2$ \\
\hline Ingresos reducidos y problemas económicos & $30.33 \%$ & $19.73 \%$ \\
\hline Reducción o pérdida de trabajo & $30.33 \%$ & $7.20 \%$ \\
\hline Cambios en la dinámica familiar & $39.32 \%$ & $73.06 \%$ \\
\hline
\end{tabular}


En la tabla 4 se puede observar el cambio registrado en el segundo periodo, en el que la afectación más significativa es la modificación en la dinámica familiar al tener, a decir de los docentes, la necesidad de conjugar el trabajo con la educación de los hijos y las labores del hogar. Los ingresos reducidos y problemas económicos de un periodo a otro, así mismo, en menor grado, ya que la afectación se redujo en $23 \%$, se presentó la reducción o pérdida de trabajo para este colectivo en el segundo semestre.

Tabla 5: Dificultades que enfrentan los docentes durante la pandemia por Covid-19

\begin{tabular}{|l|l|}
\hline \multicolumn{1}{|c|}{ Docentes 2020/1 } & \multicolumn{1}{c|}{ Docentes 2020/2 } \\
\hline $\begin{array}{l}\text { 1. La desinformación y exceso de noticias } \\
\text { basura (fake news) }\end{array}$ & 1. Acostumbrarse al trabajo desde su casa \\
\hline 2. Cambio de planes & $\begin{array}{l}\text { 2. La ansiedad y estrés por la incertidumbre } \\
\text { económica y laboral }\end{array}$ \\
\hline 3. Acostumbrarse al trabajo desde su casa & 3. Cambio de planes \\
\hline $\begin{array}{l}\text { 4. La ansiedad y estrés por la } \\
\text { incertidumbre económica y laboral }\end{array}$ & $\begin{array}{l}\text { 4. Incremento en los costos de necesidades } \\
\text { básicas }\end{array}$ \\
\hline $\begin{array}{l}\text { 5. Incremento en los costos de } \\
\text { necesidades básicas }\end{array}$ & $\begin{array}{l}\text { 5. La desinformación y exceso de noticias } \\
\text { basura (fake news) }\end{array}$ \\
\hline
\end{tabular}

En el primer periodo 2020/1 los docentes plantearon que la desinformación y exceso de noticias basura fue la principal dificultad que enfrentaron; siendo esta misma dificultad para el siguiente periodo de 2020/2 como la dificultad que menos les afectó. Para el semestre de agosto-diciembre de 2020 los docentes plantearon que la principal dificultad que encararon fue acostumbrarse al trabajo desde su casa, seguido de la ansiedad y estrés por la incertidumbre económica y laboral. Otros resultados relevantes plantean la percepción de los docentes vinculada con su satisfacción en torno a la impartición de cátedra a través de la modalidad virtual (Fig.1), así como lo que consideran que aprenden los estudiantes en aquélla (Figura 2).

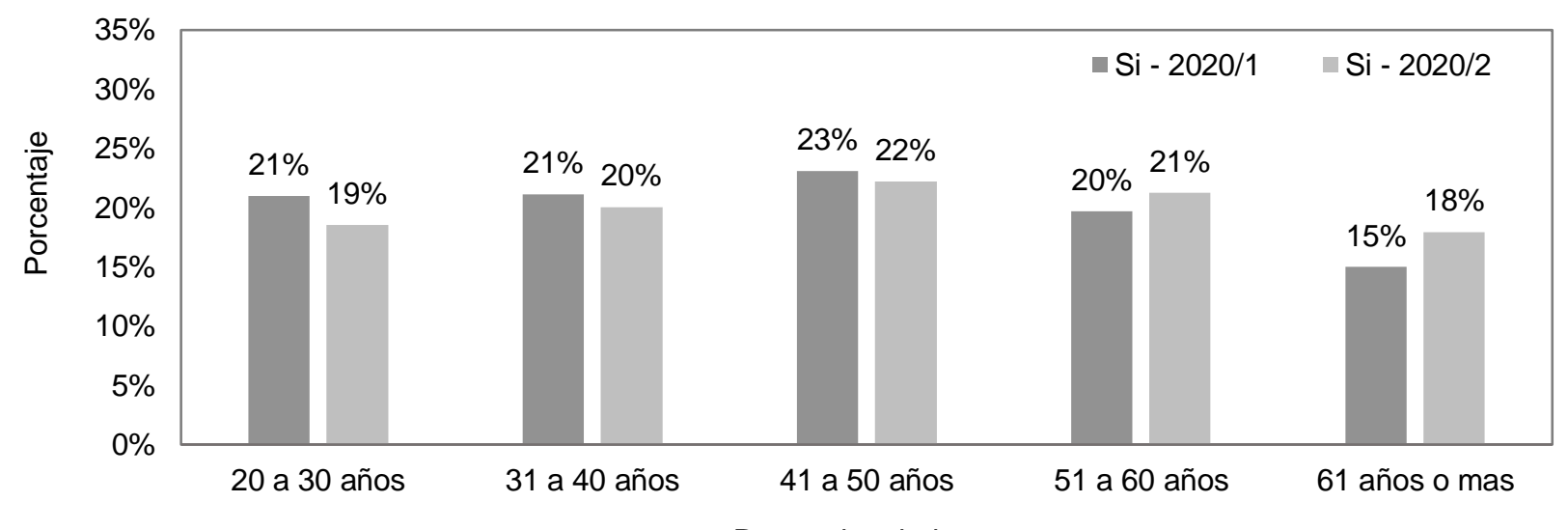

Rango de edades

Fig. 2: Es de su agrado impartir clases en modalidad virtual

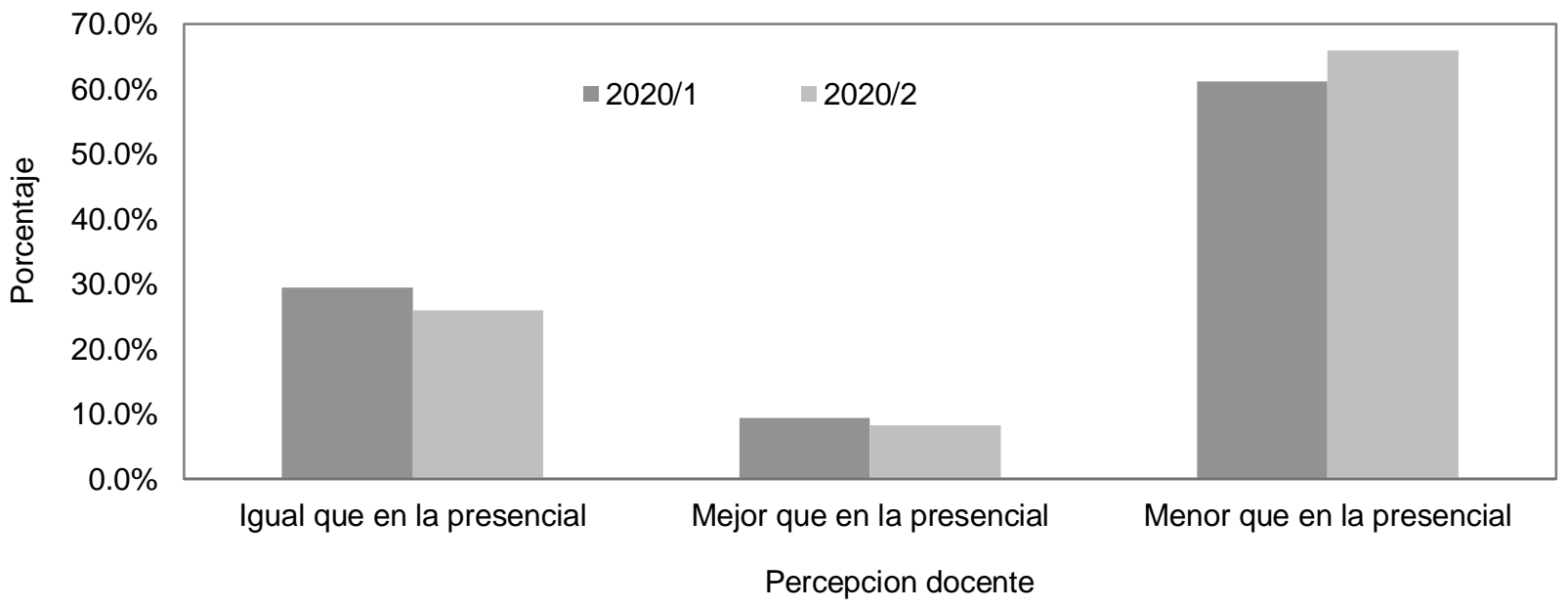

Fig. 3: En la educación virtual considera que los alumnos aprenden 
Aún y cuando los docentes manifiestan su gusto por la educación virtual (Fig.1), un porcentaje elevado, más de $60 \%$ en ambos periodos no consideran que los estudiantes logran el mismo aprendizaje que en la modalidad presencial (Fig. 2). Otro aspecto relevante se presenta en cuando a la comparación entre el primer y segundo semestres del año 2020 con respecto a la experiencia en el manejo de la plataforma y la percepción de los profesores (Tabla 6) sobre la modificación de sus competencias en TIC en el citado periodo. En la Tabla 7 se presentan los resultados sobre las competencias en TIC que consideran tener cada uno de los colectivos analizados.

Tabla 6: Nivel de experiencia en el manejo de la plataforma digital Moodle

\begin{tabular}{|l|c|c|c|}
\hline & $2020 / 1$ & $2020 / 2$ & $\begin{array}{c}\text { Valor } \\
\mathrm{P}\end{array}$ \\
\hline Avanzado & $16.00 \%$ & $18.30 \%$ & \\
\cline { 1 - 3 } Intermedio & $32.00 \%$ & $57.40 \%$ & \multirow{2}{*}{$2.21111^{\mathrm{e}-19}$} \\
\hline Principiante & $38.00 \%$ & $22.20 \%$ & \\
\cline { 1 - 3 } No sé utiliza la plataforma digital Moodle & $0.14 \%$ & $0.02 \%$ & \\
\hline
\end{tabular}

Tabla 7: Percepción de la evolución en el dominio del manejo de las TIC

\begin{tabular}{|c|c|c|c|c|}
\hline & $\begin{array}{l}\text { Media } \\
\text { Docentes } \\
2020 / 1\end{array}$ & $\begin{array}{l}\text { Media } \\
\text { Docentes } \\
2020 / 2\end{array}$ & $\begin{array}{l}\text { Media } \\
\text { Global }\end{array}$ & $\begin{array}{l}\text { Valor } \\
\mathrm{p}\end{array}$ \\
\hline \multicolumn{5}{|l|}{ Básicas } \\
\hline Maneja efectivamente el Word, Excel, PowerPoint y otros programas & 4.17 & 4.15 & 4.16 & 0.897 \\
\hline Maneja Internet & 4.38 & 4.33 & 4.35 & 0.534 \\
\hline Sabe utilizar la plataforma de la universidad (Moodle) & 3.12 & 3.72 & 3.42 & 0.000 \\
\hline \multicolumn{5}{|l|}{ Aplicación } \\
\hline Utiliza herramientas digitales para sus presentaciones académicas & 3.85 & 3.96 & 3.91 & 0.165 \\
\hline $\begin{array}{l}\text { Para elaborar material didáctico y preparar mis clases realizo } \\
\text { búsquedas de información con Google }\end{array}$ & 3.85 & 3.94 & 3.90 & 0.231 \\
\hline $\begin{array}{l}\text { Para elaborar material didáctico y preparar mis clases realizo } \\
\text { búsquedas de información con Google académico }\end{array}$ & 3.74 & 3.77 & 3.76 & 0.600 \\
\hline \multicolumn{5}{|l|}{ Profundización } \\
\hline Tiene habilidad para resolver problemas utilizando las TIC & 3.70 & 3.74 & 3.72 & 0.206 \\
\hline $\begin{array}{l}\text { Tiene las habilidades y el conocimiento que le permiten realizar un } \\
\text { proyecto de investigación utilizando las TIC }\end{array}$ & 3.73 & 3.79 & 3.76 & 0.524 \\
\hline $\begin{array}{l}\text { Tiene habilidades para generar preguntas clave y problemas, } \\
\text { formulándolos con claridad y precisión }\end{array}$ & 3.98 & 3.95 & 3.97 & 0.715 \\
\hline \multicolumn{5}{|l|}{ Trabajo Colaborativo y Aprendizaje Permanente } \\
\hline Encarga trabajos académicos en equipo por medios digitales & 3.38 & 3.50 & 3.44 & 0.370 \\
\hline $\begin{array}{l}\text { Está abierta/o a formas de pensamiento diferentes a la suya que le } \\
\text { permita enriquecer el conocimiento }\end{array}$ & 4.59 & 4.63 & 4.61 & 0.514 \\
\hline Utiliza las TIC para aprender y enseñar de manera permanente & 3.84 & 4.08 & 3.96 & 0.008 \\
\hline \multicolumn{5}{|l|}{ Éticas } \\
\hline $\begin{array}{l}\text { Al elaborar material didáctico y apuntes siempre cita al autor o autores } \\
\text { que escribieron los documentos que utilizó como referencia, ya sea de } \\
\text { manera textual o de parafraseo }\end{array}$ & 4.11 & 4.09 & 4.10 & 0.747 \\
\hline $\begin{array}{l}\text { Utiliza las TIC para aprender de otros y reconocerles su trabajo al } \\
\text { citarles }\end{array}$ & 4.06 & 4.11 & 4.08 & 0.109 \\
\hline
\end{tabular}

Los resultados generales arrojan que los docentes se consideran más competentes en cuanto al manejo de TIC y uso de la plataforma Moodle, en relación del periodo a los dos periodos analizados; se observa una diferencia significativa $(p<.000)$ relacionada con la utilización de la plataforma digital Moodle utilizada por la universidad. 


\section{DISCUSIÓN}

La presente investigación analiza la percepción de docentes universitarios de la $\mathrm{UACH}$, sobre su quehacer académico y personal durante la contingencia de la COVID-19, a partir del planteamiento de las hipótesis que enseguida se enuncian: Sí existen diferencias significativas entre la percepción del primer y del segundo semestre del 2020 de los docentes de la $\mathrm{UACH}$, en torno a los efectos del Covid-19 en su familia y sobre el aprendizaje en la educación en línea.

Los efectos del Covid-19 en su familia, esta hipótesis se comprueba parcialmente, ya que hubo una modificación entre el orden de mayor afectación, siendo este el cambio en la dinámica familiar el aspecto de mayor incidencia, ya que pasó de $39.32 \%$ a $73.06 \%$, este dato en el cual un porcentaje elevado de docentes comenta que su familia se afectó a través del aislamiento, la reducción de ingreso y pérdida de empleo, así como la modificación de la dinámica familiar, misma que al vincularse con la Teoría de la Auto-complejidad (Linville, 1985) la cual establece que los individuos necesitan realizar diversos roles sociales como educación, ocio, y familia, en su propio contexto; queda a la deriva, ya que, debido a la pandemia estos procesos sociales se han llevado a cabo en un mismo espacio, el hogar. Haciendo eco de Díaz Barriga (2020, pág. 19), el espacio educativo se ha perdido y, de acuerdo con Ruiz (2020), todo este desafío puede llevar a dejar de reconocer que el mayor riesgo es dejar de contemplar las condiciones de emergencias (sanitarias, sociales, psicológicas y tecnológicas) generadas por la pandemia del Covid-19, ya que ello afectará a la educación y sus implicancias formativas para el desarrollo humano; lo que lleva a plantear la importancia de realizar estudios que profundicen en abordar los pensamientos y sentimientos de los maestros sobre la enseñanza remota, así como las necesidades laborales y los recursos que definen la nueva realidad en el contexto del covid-19 (Sokal et al., 2020).

Así mismo, el nivel de experiencia en el uso de la plataforma Moodle se incrementó entre los dos periodos. Las competencias básicas, de aplicación, profundización, trabajo colaborativo, éticas y de aprendizaje para toda la vida utilizando las TIC se modificaron y presentan una diferencia significativa entre un semestre y otro entre los docentes universitarios. Cabe destacar que todas las competencias en TIC mejoraron su calificación entre el primer y el segundo semestre del 2020; sin embargo, sólo la diferencia de medias resultó significativa en la utilización de la plataforma Moodle. Esto lleva a rechazar la hipótesis que plantea una diferencia significativa entre la comparación de las competencias de los docentes de un semestre a otro. De hecho, en lo que concierne a las competencias de aplicación y profundización, los catedráticos se han calificado por debajo del 4, lo que indica un espacio para la mejora y en el uso de la plataforma. Estos resultados llevan a plantear que los profesores necesitan fortalecer sus competencias en TIC al tener un dominio más avanzado para promover el aprendizaje en línea, como una alternativa a la enseñanza presencial (Jogezai , et al., 2021) y que puedan transferir el conocimiento que redunde en el desarrollo estratégico y crítico del uso responsable y seguro de los alumnos hacia las tecnologías digitales. (Álvarez-Flores, 2021).

El aprendizaje en la educación en línea, desde la percepción de los docentes. Entre los hallazgos, destaca el hecho de que los docentes consideran que los estudiantes aprenden mejor en la educación presencial, de cara a cara, aspecto que concuerda con lo que han planteado que los estudiantes que no han utilizado las tecnologías educativas de manera constante tienen menor percepción de su eficacia en el aprendizaje, por lo que se considera que el uso de nuevas tecnologías educativas, de no utilizarse adecuadamente, pueden actuar en detrimento del proceso de enseñanza aprendizaje (Aguilera-Hermida, 2020), lo que concuerda con los resultados de la investigación realizada por Oyedotun (2020), quien indica que hay menor participación entre alumnos y maestros, a decir de algunos, los alumnos ya no participan en el debate de clase como lo hacen en la clase tradicional cara a cara y, a menudo, hay poca o ninguna retroalimentación cuando se hacen preguntas; sin embargo, se ha avanzado en entrenamiento a las docentes y estudiantes en torno a la educación digital y se comparte material y videos que benefician el proceso de enseñanza-aprendizaje. Sobre las dificultades encaradas por los docentes a raíz de la pandemia para el semestre de agosto-diciembre de 2020 los docentes plantearon que la principal dificultad que encararon fue acostum-brarse al trabajo desde casa, seguido de la ansiedad y estrés por la incertidumbre económica y laboral.

\section{CONCLUSIONES}

La pandemia provocada por la COVID-19 es un acontecimiento que ha marcado la vida de las personas en todos los aspectos sobre todo en la forma en como venían viviendo sus vidas; no se puede dejar de lado alguno de los aspectos que hemos venido analizando puesto que todos forman parte del quehacer diario de Ios individuos. Las IES como parte del Sistema Educativo en todos los países y los actores que forman parte de este proceso formativo, deben buscar siempre estar aprendiendo de las situaciones y circunstancias, derivando políticas y estrategias con las cuales puedan dar la atención que requieren tanto alumnos como docentes, lo anterior con el firme propósito de que puedan desarrollar todas las competencias requeridas para alcanzar a desarrollar de manera correcta los perfiles de egreso requeridos para hacer frente al mercado laboral 


\section{NOTACIÓN}

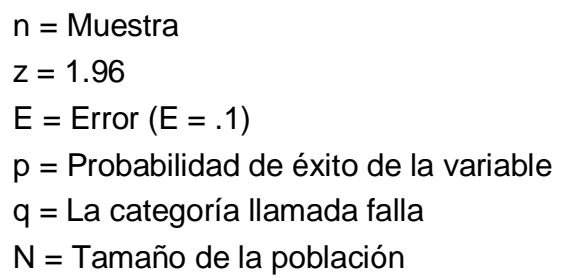

\section{REFERENCIAS}

Arras-Vota, A. D., Torres-Gastelú, C. A., \& García-Valcárcel, A., Students' Perceptions About Their Competencies in Information and Communication Technologies (ICTs), https://doi:10.4185/RLCS-66-2011-927-130-152, Revista Latina de Comunicación Social, 66, 1-26 (2011)

Aguilera-Hermida, P., College students' use and acceptance of emergency online learning due to COVID-19, https://doi.org/10.1016/j.jedro.2020.100011, International Journal of Educational Research Open, 1, 1-8 (2020)

Alvarez-Flores, E. P., Uso crítico y seguro de tecnologías digitales de profesores universitarios, https://dx.doi.org/10.4067/S0718-50062021000100033, Formación universitaria, 14, 33-44 (2021)

Arriagada, P., Pandemia Covid-19: educación a distancia. O las distancias en la educación, Revista Internacional de Educación para la Justicia Social, 9, 1-3, (2020)

Cendon, E. Lifelong Learning at Universities: Future Perspectives for Teaching and Learning, https:// doi.org/10.7821/naer.2018.7.320, Journal of New Approaches in Educational Research, 7(2), 81-87 (2018)

Cervantes, E., Gutiérrez, P., Resistir la Covid-19. Intersecciones en la Educación de Ciudad Juárez, México, Revista Internacional de Educación para la Justicia Social, 9, 1-23, (2020)

Díaz-Barriga, A., La escuela ausente, la necesidad de replantear su significado. En IISUE, Educación y pandemia, una visión académica, Instituto de Investigaciones sobre la Universidad y la Educación de la UNAM,19-29, (2020)

Eirín-Nemiña, R. Las Comunidades de Aprendizaje como Estrategia de Desarrollo Profesional de Docentes de Educación Física, http://dx.doi.org/10.4067/S0718-07052018000100259, Estudios Pedagógicos, XLIV (1), 259-278 (2018)

Frías, D. (2014). Alfa de Cronbach y consistencia interna de los ítems de un instrumento de medida. Recuperado de http://www.uv.es/friasnav/ApuntesSPSS.pdf

Galindo, R., Galindo, L. y otros cuatro autores, Acercamiento Epistemológico a la Teoría del Aprendizaje Colaborativo, Apertura, e-ISSN: 2007-1094, 4(2), 156-169, (2013)

Infante, J. C., Gallardo, J., \& Infante, A., The Importance of ICTs for Students as a Competence for their Future Professional Performance: The Case of the Faculty of Business Studies and Tourism of the University of Huelva Alfonso, https://doi:10.7821/naer.2019.7.434, Journal of New Approaches in Educational Research, 8(2), 201-213 (2019)

Jaramillo, P., Cristina, H., \& Rincón, Y., ¿Cómo Manejan Información los Estudiantes de Educación Superior? El Caso de la Universidad de La Sabana, Colombia. Información, Cultura y Sociedad, ISSN 1851-1740, 117-143 (2011)

Jogezai, N., Baloch, F. y otros 4 autores, Teachers' attitudes towards social media (SM) use in online learning amid the COVID-19 pandemic: the effects of SM use by teachers and religious scholars during physical distancing, https://doi.org/10.1016/j.heliyon.2021.e06781, Heliyon, 7, 1-9 (2021)

Landero, R. y González, M. (2006). Estadística con SPSS y metodología de la investigación. Ciudad de México, México: Trillas.

Linville, P. W., Self-complexity and affective extremity: Don't put all of your eggs in one cognitive basket, https://doi.org/10.1521/soco.1985.3.1.94, Social Cognition, 3, 94-120, (1985)

Lomborg, S., Personal Internet Archives and Ethics, https://doi:10.1177/1747016112459450, Research Ethics, 9(1), 20$31(2012)$

Marshall, K., Has Technology Introduced New Ethical Problems, https://doi.org/10.1023/A:1006154023743, Journal of Bussines Ethics, 19(1), 81-90 (1999)

Moreno, M. A., Nelly, E., \& Garcia, D. J. Percepción de los Estudiantes de Enfermería sobre el Ambiente de Aprendizaje durante sus Prácticas Clínicas. Cuidarte, ISSN: 2216-0973, 4(1), 444-449 (2013)

Maryuningsih, Y., Hidayat, T. y otros dos autores, Profile of Information and Communication Technologies (ICT) Skills of Prospective Teachers, https://doi:10.1088/1742-6596/1521/4/042009, Journal of Physics: Conference Series, 1521, 1-8 (2019)

Martzoukou, K., Fulton, C. y otros dos autores, A Study of Higher Education Students Self-Perceived Digital Competences for Learning and Everyday Life Online Participation, https://doi.org/10.1108/JD-03-2020-0041, Journal of Documentation, 1-51 (2020) 
Muñoz, J. L., \& Lluch, L., Educación y Covid-19: Colaboración de las Familias y Tareas Escolares, Revista Internacional de Educación para la Justicia Social, 9(3), 1 - 17, (2020)

Olivia, N., Casanovas, M., \& Capdevila, Y., Academic Writing and the Internet: Cyber-Plagiarism amongst University Students, https://doi:10.7821/naer.2019.7.407, Journal of New Approaches in Educational Research, 8(2), 112-125. (2019)

Oyedotun T., D., Sudden change of pedagogy in education driven by COVID-19: Perspectives and evaluation from a developing country, https://doi.org/10.1016/j.resglo.2020.100029, Research in Globalization, 2, 1-5, (2020)

Ruiz, G., Marcas de la Pandemia: El Derecho a la Educación afectado, https://doi.org/10.15366/riejs2020.9.3.003, Revista Internacional de Educación para la Justicia Social, 9(3), 45 - 59, (2020)

Hernández-García S., Chaparro Peláez J. \& Prieto, J. L., Emergency Remote Teaching and Students' Academic Performance in Higher Education during the COVID-19 Pandemic: A Case Study, https://doi.org/10.1016/j.chb.2021.10671, Computers in Human Behavior, 119, 1-18, (2021)

Sokal, L., Eblie L. \& Babb J., Canadian teachers' attitudes toward change, efficacy, and burnout during the COVID-19 pandemic, https://doi.org/10.1016/j.ijedro.2020.100016, International Journal of Educational Research Open, 1, 1-8, (2020)

Torrecillas, C., El reto de la docencia online para las universidades públicas españolas ante la pandemia del Covid-19, https://www.ucm.es/, ICEI Papers, 16, 1-4, (2020)

Universidad Autónoma de Chihuahua. (08 de 04 de 2020). Historia

Yin, R., Case Study Research Design and Methods. London U.K.: Thousand Oaks: Sage Publications (2009)

Zambrano, C., Albarrán, F., \& Salcedo, P. A., Percepción de Estudiantes de Pedagogía Respecto de la Autorregulación del Aprendizaje, Percepción de Estudiantes de Pedagogía Respecto de la Autorregulación del Aprendizaje, http://dx.doi.org/10.4067/S0718-50062018000300073, Formación Universitaria, 11(3), 73-86 (2018) 